\title{
miRNA transported by exosomes: a key machinery in tumor microenvironment mediated chemoresistance
}

\author{
Teresa Gagliano, Thomas Simon, Georgios Giamas \\ Department of Biochemistry and Biomedicine, School of Life Sciences, University of Sussex, Brighton, BN1 9QG, UK \\ Correspondence to: Teresa Gagliano. Department of Biochemistry and Biomedicine, School of Life Sciences, University of Sussex, Falmer, Brighton, \\ BN1 9QG, UK. Email: T.Gagliano@sussex.ac.uk. \\ Comment on: Au Yeung CL, Co NN, Tsuruga T, et al. Exosomal transfer of stroma-derived miR21 confers paclitaxel resistance in ovarian cancer cells \\ through targeting APAF1. Nat Commun 2016;7:11150.
}

Submitted Nov 23, 2016. Accepted for publication Nov 25, 2016.

doi: $10.21037 /$ tcr.2016.12.40

View this article at: http://dx.doi.org/10.21037/tcr.2016.12.40

It is well established that the tumor microenvironment (TME) actively participates in fundamental processes such as tumor initiation, invasion and response to therapies, apart from its documented scaffold role. Ultimately, the TME can be defined as a tri-dimensional network structured by the tumor-associated extra-cellular matrix (ECM) in which tumor cells interact with various types of stromal cells in an attempt to hijack the normal organization of the host tissue and support cancer growth.

The TME has various aptitudes that can induce both beneficial and adverse consequences for tumorigenesis. Microenvironment components can use different way to influence the behavior of tumor cells including: (I) signaling induced by ECM components at the tumor cells' surface; (II) remodeling of the extracellular matrix (1); (III) signal transmission through cytokines secretion (2); (IV) modulation of tumor cell signaling and gene expression via extracellular vesicles (EVs) content (3).

Among the components of the TME, fibroblasts represent the main cellular type, while the latest evidence suggests that the key communication option for cancer-associated fibroblasts (CAFs) is mediated via exosomes secretion (4).

Exosomes are defined as lipoprotein shells that contain lipids, proteins and nucleic acids [mRNA, microRNAs (miRNAs)] with a diameter ranging between 20 and $120 \mathrm{~nm}$ (4). Exosomes can carry their content from one cell to another for far distances, mainly through blood, protecting it from the degrading activity of extracellular enzymes and then allow the emitting cell to directly influence the recipient cell. Their production and secretion is increased in cancer cells compared to normal cells.
In addition, their molecular composition is very wide, and could be significantly vary depending on cell type, conditions ( $\mathrm{pH}$, hypoxia) and treatment; for these reasons, their role in the TME has been recently considered.

Regarding the proteomic content of exosomes, recent studies have reported that exosomes containing amphiregulin can contribute to cancer invasion and metastasis in colorectal cancer (5). Metalloproteases, proteins able to degrade extracellular matrix proteins, can also be incorporated within exosomes and correlate with cancer invasion. Exosomes from leukemic and glioblastoma cells have been shown to increase endothelial cells' tube formation and vascularization by increasing angiogenesis through their cargo proteins $(6,7)$.

Despite the importance of the proteomic content of exosomes, the significance of miRNAs as components of exosomes has also been investigated and linked to various diseases including cancer. miRNAs exist organically as the most biologically stable nucleic acid comprising 19-23 nucleotides that can act as post-transcriptional modulators of gene expression via miRNA-mRNA interactions. The majority of published papers report the effects of miRNAs on tumor cells, but another interesting point of view suggests that they can be involved in fibroblasts transformation into CAFs. Currently, several studies have suggested exosome-mediated miRNAs transduction plays a pivotal role in the crosstalk between tumors cells and the TME. Thus, it has been recently demonstrated that tumor cell derived-exosomal miR-9 is able to promote fibroblasts motility and transformation into CAFs, enhancing breast cancer invasion (8). Moreover, overexpression of miR-21 has been implicated in different tumor types including lung, 


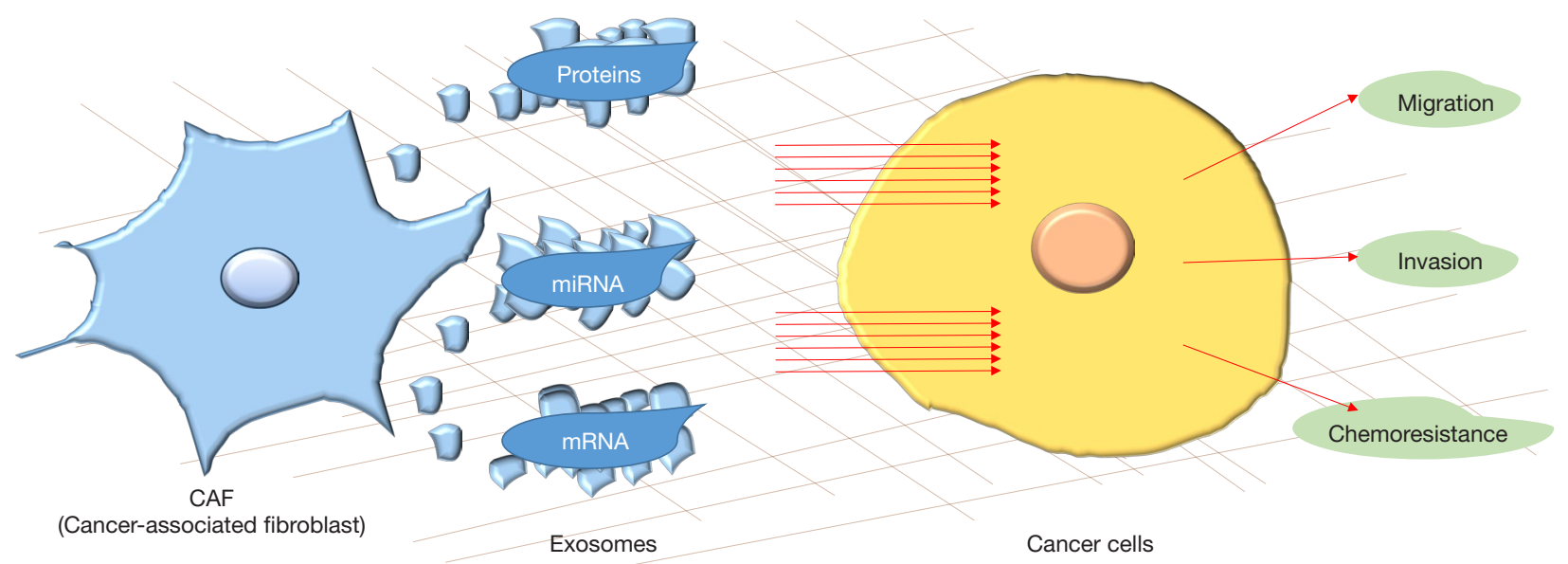

Figure 1 Schematic representation of cancer-associated fibroblast (CAF) secreted exosomes and their effects on cancer cells. CAFs can influence tumor cells by different mechanisms. Exosomes secretion is currently considered as the main machinery used by CAFs to influence tumor cells behavior. Exosomes from CAFs can transport proteins, mRNAs, and miRNAs, transfer of these molecules can lead to increased migration, invasion and chemoresistance of tumor cells.

ovarian, breast, colon, gastric, glioblastoma and pancreatic. miR-21 is one of the most abundant miRNAs in exosomes and is considered to be an oncogene, as it promotes tumor cell proliferation, migration, and invasiveness by targeting a number of tumor-suppressor genes and exhibiting proangiogenic properties (9). Due to its upregulation in cancers and oncogenic properties, miR-21 plasma levels have been used as markers for several tumors types (10) (Figure 1).

Au Yeung et al. (11) have shown that miR-21, transferred via exosomal vesicles from CAFs and cancer associated adipocytes, can confer chemoresistance to ovarian cancer cells by modulating the apoptotic protease-activating factor 1 (APAF1) and the formation of apoptosome while interfering with the recruitment and activation of cellkilling caspases, a mechanism that has already been related with drug resistance (12).

This work highlighted the pivotal role of interactions between tumor cells and cancer-associated cells in the TME to support invasion and resistance to therapies. On the other hand, the authors used type I collagen matrix to study the invasion of cancer cells transfected or not with miR21. Unfortunately, those cancer cells have not been co-cultured with CAFs or adipocytes, which would have allowed the study of cancer cells interactions with stromal counterparts in a tridimensional matrix. An in vivo mouse model was used instead, where cancer cells have been co-injected with mouse embryonic fibroblasts. In this setting TME cannot be directly manipulated and, consequently, intercellular relationship between tumor and stromal cells cannot be clearly assessed.

An in vivo mouse model was used instead, allowing the authors to translate their results to a model incorporating the TME. Mouse embryonic fibroblasts have been used in this in vivo model to study the CAF/ovarian cancels interactions, even if the authors observed the highest amount of miR21 in CAA-derived exosomes. Deciphering $\mathrm{CAA}$ /ovarian cancer cells interactions via exosomes would actually help to understand the set-up of the premetastatic niche in which tumor cells-derived exosomes play an important role (13). Thus, it would be also interesting to describe changes in the content and amount of exosomes produced by CAA and CAF in response to paclitaxel.

Interestingly, it has been demonstrated that miR-21 is able to promote prostate cell neoplastic transformation while in clear-cell renal carcinoma it acts as an oncogenic factor by increasing cell proliferation and epithelialmesenchymal transition $(14,15)$. The effects of miR-21 transported in CAF exosomes on normal ovarian cells have not been tested. It would be interesting to asses if miR-2 1 could drive a possible CAF mediate tumorigenesis. These additional data would provide a complete framework of the consequences of chemotherapies on TME, shedding light on the origins of tumor resistance to treatments (Table 1).

Overall miRNAs appear to represent one of the most powerful content of exosomes, particularly of those secreted 
Table 1 miRNA related to tumor drug resistance

\begin{tabular}{llll}
\hline miRNA & Drug & Target/mechanism & Tumor \\
\hline miR-21 & Paclitaxel, temozolomide & APAF1/caspase-3, apoptosis & Ovarian cancer, glioblastoma \\
miR-196a-5p & Gentamicine & Apoptosis & Bladder cancer \\
miR-221 & 5-FU & DKK2 & Esophageal, adenocarcinoma \\
miR-645 & Cisplatin & Apoptosis & Osteosarcoma \\
\hline
\end{tabular}

Several miRNAs have been associated with chemoresistance. The table shows examples of miRNAs (either transported by exosomes or directly secreted by tumor cells) implicated in this process including drugs, signaling pathways and tumor type information. miRNAs, microRNAs.

by TME. Interestingly, miR-21 is not the only miRNA that has been associated with chemoresistance either positively or negatively. miR-645 has been involved in cisplatin-induced apoptosis in osteosarcoma (16), while miR-196a-5p has been shown to have a role in bladder cancer gentamicinemediated apoptosis (17). miR-221 has been associated in 5-FU resistance of esophageal adenocarcinoma (18). An important role in cell cycle control of pituitary cells is played by miR-26a and related pathways (19). miR-122 derived from cancer exosomes, has been shown to be able to enhance energetic metabolism and to increase cancer progression (20). miRNAs found in exosomes have not only been related to chemoresistance but also with other crucial physio/ pathologic processes: miR-15-16 expression has been found in exosomes from different type of vascular cells, displaying antiangiogenic activity targeting VEGF-A, while miR-200 family has been associated with cell adhesion (8).

The influence of TME and the mechanism behind it have opened a new path to be considered in drug design and tumor treatment. Stroma cell types in the TME are relatively stable particularly when compared to tumor cells, and for this reason they are currently a putative therapeutic target which can reduce the risk of resistance and tumor recurrence. The increasing knowledge of TME/miRNA/ exosomes systems and tumor/TME cross-talk will hopefully lead to a better clinical managing of cancer patients.

\section{Acknowledgments}

Funding: None.

\section{Footnote}

Provenance and Peer Review: This article was commissioned and reviewed by the Section Editor Xiaoying Zhou (Institute of Gastroenterology, the First Affiliated Hospital of Nanjing
Medical University, Nanjing, China).

Conflicts of Interest: All authors have completed the ICMJE uniform disclosure form (available at http://dx.doi. org/10.21037/tcr.2016.12.40). The authors have no conflicts of interest to declare.

Ethical Statement: The authors are accountable for all aspects of the work in ensuring that questions related to the accuracy or integrity of any part of the work are appropriately investigated and resolved.

Open Access Statement: This is an Open Access article distributed in accordance with the Creative Commons Attribution-NonCommercial-NoDerivs 4.0 International License (CC BY-NC-ND 4.0), which permits the noncommercial replication and distribution of the article with the strict proviso that no changes or edits are made and the original work is properly cited (including links to both the formal publication through the relevant DOI and the license). See: https://creativecommons.org/licenses/by-nc-nd/4.0/.

\section{References}

1. Jolly LA, Novitskiy S, Owens P, et al. FibroblastMediated Collagen Remodeling Within the Tumor Microenvironment Facilitates Progression of Thyroid Cancers Driven by BrafV600E and Pten Loss. Cancer Res 2016;76:1804-13.

2. Cirillo N, Hassona Y, Celentano A, et al. Cancerassociated fibroblasts regulate keratinocyte cell-cell adhesion via TGF- $\beta$-dependent pathways in genotypespecific oral cancer. Carcinogenesis 2016. [Epub ahead of print].

3. Wendler F, Favicchio R, Simon T, et al. Extracellular vesicles swarm the cancer microenvironment: from tumorstroma communication to drug intervention. Oncogene 
2016. [Epub ahead of print].

4. Wendler F, Stamp GW, Giamas G. Tumor-stromal cell communication: small vesicles signal big changes. Trends in Cancer 2016;2:326-9.

5. Higginbotham JN, Demory Beckler M, Gephart JD, et al. Amphiregulin exosomes increase cancer cell invasion. Curr Biol 2011;21:779-86.

6. Endres M, Kneitz S, Orth MF, et al. Regulation of matrix metalloproteinases (MMPs) expression and secretion in MDA-MB-231 breast cancer cells by LIM and SH3 protein 1 (LASP1). Oncotarget 2016. [Epub ahead of print].

7. Mineo M, Garfield SH, Taverna S, et al. Exosomes released by K562 chronic myeloid leukemia cells promote angiogenesis in a Src-dependent fashion. Angiogenesis 2012;15:33-45.

8. Baroni S, Romero-Cordoba S, Plantamura I, et al. Exosome-mediated delivery of miR-9 induces cancerassociated fibroblast-like properties in human breast fibroblasts. Cell Death Dis 2016;7:e2312.

9. Lopatina T, Gai C, Deregibus MC, et al. Cross talk between cancer and mesenchymal stem cells through extracellular vesicles carrying nucleic acids. Front Oncol 2016;6:125.

10. Fernandez-Mercado M, Manterola L, Larrea E, et al. The circulating transcriptome as a source of non-invasive cancer biomarkers: concepts and controversies of noncoding and coding RNA in body fluids. J Cell Mol Med 2015;19:2307-23.

11. Au Yeung CL, Co NN, Tsuruga T, et al. Exosomal transfer of stroma-derived miR2 1 confers paclitaxel resistance in ovarian cancer cells through targeting APAF1. Nat Commun 2016;7:11150.

12. Zatelli MC, Gagliano T, Pelà M, et al. N-carbamidoyl-

Cite this article as: Gagliano T, Simon T, Giamas G. miRNA transported by exosomes: a key machinery in tumor microenvironment mediated chemoresistance. Transl Cancer Res 2016;5(Suppl 7):S1479-S1482. doi: 10.21037/tcr.2016.12.40 4-((3-ethyl-2,4,4-trimethylcyclohexyl)methyl)benzamide enhances staurosporine cytotoxic effects likely inhibiting the protective action of Magmas toward cell apoptosis. J Med Chem 2014;57:4606-14.

13. Costa-Silva B, Aiello NM, Ocean AJ, et al. Pancreatic cancer exosomes initiate pre-metastatic niche formation in the liver. Nat Cell Biol 2015;17:816-26.

14. Sánchez CA, Andahur EI, Valenzuela R, et al. Exosomes from bulk and stem cells from human prostate cancer have a differential microRNA content that contributes cooperatively over local and pre-metastatic niche. Oncotarget 2016;7:3993-4008.

15. Cao J, Liu J, Xu R, et al. MicroRNA-21 stimulates epithelial-to-mesenchymal transition and tumorigenesis in clear cell renal cells. Mol Med Rep 2016;13:75-82.

16. Wang Y, Zhang L, Zheng X, et al. Long non-coding RNA LINC00161 sensitises osteosarcoma cells to cisplatininduced apoptosis by regulating the miR-645-IFIT2 axis. Cancer Lett 2016;382:137-46.

17. Pan J, Li X, Wu W, et al. Long non-coding RNA UCA1 promotes cisplatin/gemcitabine resistance through CREB modulating miR-196a-5p in bladder cancer cells. Cancer Lett 2016;382:64-76.

18. Wang Y, Zhao Y, Herbst A, et al. miR-221 mediates chemoresistance of esophageal adenocarcinoma by direct targeting of DKK2 expression. Ann Surg 2016;264:804-14.

19. Gentilin E, Tagliati F, Filieri C, et al. miR-26a plays an important role in cell cycle regulation in ACTH-secreting pituitary adenomas by modulating protein kinase $\mathrm{C} \delta$. Endocrinology 2013;154:1690-700.

20. Fong MY, Zhou W, Liu L, et al. Breast-cancer-secreted miR-122 reprograms glucose metabolism in premetastatic niche to promote metastasis. Nat Cell Biol 2015;17:183-94. 